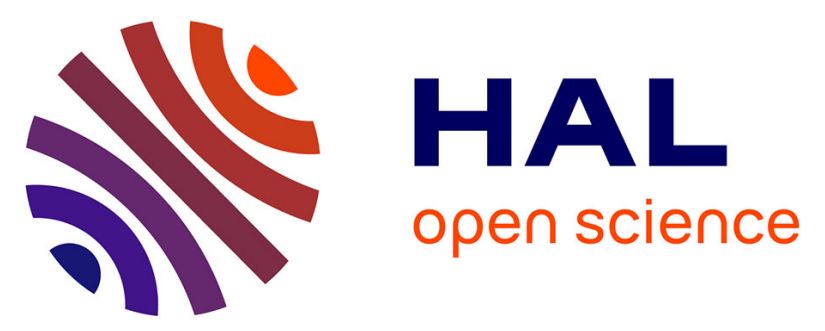

\title{
Claudin-1, miR-122 and apolipoprotein E transductions improve the permissivity of SNU-182, SNU-398 and SNU-449 hepatoma cells to hepatitis C virus
}

Carole Fournier, Thomas Walter Hoffmann, Virginie Morel, Véronique Descamps, Jean Dubuisson, Etienne Brochot, Catherine François, Gilles Duverlie, Sandrine Castelain, Francois Helle

\section{To cite this version:}

Carole Fournier, Thomas Walter Hoffmann, Virginie Morel, Véronique Descamps, Jean Dubuisson, et al.. Claudin-1, miR-122 and apolipoprotein E transductions improve the permissivity of SNU-182, SNU-398 and SNU-449 hepatoma cells to hepatitis C virus. Journal of Viral Hepatitis, 2018, 25 (1), pp.63-71. 10.1111/jvh.12767 . hal-02112514

\section{HAL Id: hal-02112514 https://hal.science/hal-02112514}

Submitted on 26 Apr 2019

HAL is a multi-disciplinary open access archive for the deposit and dissemination of scientific research documents, whether they are published or not. The documents may come from teaching and research institutions in France or abroad, or from public or private research centers.
L'archive ouverte pluridisciplinaire HAL, est destinée au dépôt et à la diffusion de documents scientifiques de niveau recherche, publiés ou non, émanant des établissements d'enseignement et de recherche français ou étrangers, des laboratoires publics ou privés. 


\section{Claudin-1, miR-122 and Apolipoprotein E}

2 transduction improves the permissivity of SNU-182,

$3 \quad$ SNU-398 and SNU-449 Hepatoma cells to Hepatitis

4

5

6 Carole Fournier ${ }^{1}$, Thomas Walter Hoffmann ${ }^{1}$, Virginie Morel $^{1}$, Véronique Descamps ${ }^{1}$,

7 Jean Dubuisson ${ }^{2}$, Etienne Brochot ${ }^{1}$, Catherine Francois ${ }^{1}$, Gilles Duverlie ${ }^{1}$, Sandrine Castelain ${ }^{1}$ 8 and Francois Helle ${ }^{1, *}$

9

Running title : Permissivity of SNU cells to HCV

\section{Virus}

Author affiliations :

${ }^{1}$ EA4294, Laboratoire de Virologie, Centre Universitaire de Recherche en Santé, Centre Hospitalier Universitaire et Université de Picardie Jules Verne, 80054 Amiens, France.

${ }^{2}$ Univ. Lille, CNRS, Inserm, CHU Lille, Institut Pasteur de Lille, U1019 - UMR 8204 - CIIL - Centre d'Infection et d'Immunité de Lille, F-59000 Lille, France

*Correspondence :

Dr. F. Helle, Laboratoire de Virologie EA4294, Centre Universitaire de Recherche en Santé, Centre Hospitalier Universitaire et Université de Picardie Jules Verne, 80054 Amiens, France.

Phone : (+33)-3-22-82-53-51

E-mail : francois.helle@u-picardie.fr 
Fournier et al. - page 2

\section{Summary}

Hepatitis $\mathrm{C}$ virus (HCV) is a human hepatotropic virus but many hepatoma cell lines are not permissive to this virus. In a previous study, we observed that SNU-182, SNU-398 and SNU449 hepatoma cell lines were non-permissive to HCV. In order to understand the nonpermissivity, we evaluated the ability of each cell line to support the different steps of HCV life cycle (entry, replication and production of infectious particles). Using retroviral pseudoparticles pseudotyped with HCV envelope proteins and recombinant HCV produced in cell culture, we observed that low level or absence of claudin-1 expression limited the viral entry process in SNU-182 and SNU-398 cells, respectively. Our results also showed that supplementation of the three cell lines with miR-122 partly restored the replication of a JFH1 HCV replicon. Finally, we observed that expression of apolipoprotein E was very low or undetectable in the three cell lines and that its ectopic expression permits the production of infectious viral particles in SNU-182 and SNU-398 cells but not in SNU-449 cells. Nevertheless, the supplementation of SNU-182, SNU-398 and SNU-449 cells with claudin-1, miR-122 and apolipoprotein E was not sufficient to render these cells as permissive as $\mathrm{HuH}-7$ cells. Thus, these cell lines could serve as cell culture models for functional studies on the role of claudin-1, miR-122 and apolipoprotein E in HCV life cycle but also for the identification of new restriction and/or dependency host factors essential for HCV infection.

Keywords : ApoE, CLDN1, Hepatitis C virus, miR-122, restriction and dependency factors.

Abbreviations: $\mathrm{HCV}$, Hepatitis C virus; HCVpp, retroviral particles pseudotyped with $\mathrm{HCV}$ envelope proteins; HCVcc, recombinant $\mathrm{HCV}$ produced in cell culture; SR-BI, Scavenger receptor class B type I; CLDN1, claudin-1; OCLN, occludin; NPC1L1, Niemann-Pick C1like 1; ApoE, Apolipoprotein E. 
Fournier et al. - page 3

\section{1 | INTRODUCTION}

Hepatitis $\mathrm{C}$ virus (HCV) is an important human pathogen. Worldwide, over 170 million people are chronically infected. Furthermore, $75 \%$ to $80 \%$ of $\mathrm{HCV}$-infected patients develop chronic infection, which may lead to cirrhosis and, ultimately, hepatocellular carcinoma. $\mathrm{HCV}$ is a small enveloped positive-strand RNA virus of the Flaviviridae family, classified in the Hepacivirus genus (1).

The study of HCV infection has long been limited by the absence of complete in vitro cell culture system. However, several alternative tools were developed in the two past decades to understand the HCV life cycle. First, the establishment of subgenomic replicons that autonomously amplify in cultured human hepatoma cells allowed to understand the mechanisms of HCV genome replication (2). Then, the generation of infectious retroviral pseudotypes displaying functional HCV glycoproteins (HCVpp) allowed for the study of HCV entry $(3,4)$. The cloning of the genotype $2 \mathrm{a}$ JFH1 strain finally paved the way for the production of infectious $\mathrm{HCV}$ virions $(\mathrm{HCVcc})$ and the investigation of the full viral cycle in human liver cancer-derived HuH-7 cells $(5,6)$. Thanks to these models, substantial progress has been made in the identification of host factors involved in HCV propagation. Among them, CD81, the scavenger receptor class B type I (SR-BI), claudin-1 (CLDN1) occludin (OCLN) and NPC1L1 were identified as important HCV entry factors (7). The liver-specific microRNA miR-122 has been shown to be one of the most important host factors for HCV replication (8). Furthermore, components of the very low density lipoprotein biosynthesis pathway such as apolipoprotein E (ApoE) have been shown to be important for the assembly and release of infectious viral particles (9-12).

The use of non-permissive cells is an interesting tool to identify new host restriction or dependency factors for viral permissivity. In a previous study, we reported that the SNU-182, SNU-398 and SNU-449 hepatocarcinoma cell lines were not able to support HCV infection 
75 (13). In this work, we evaluated the ability of each cell line to support specific steps of HCV

76 lifecycle (i.e. entry, replication and production of infectious particles) and tried to identify the host factors that prevent these cell lines from being infected by this virus.

\section{2 | MATERIALS AND METHODS}

\section{1 | Cell culture}

SNU-182 (CRL-2235), SNU-398 (CRL-2233) and SNU-449 (CRL-2234) cell lines (14) were obtained from ATCC. We also used the HuH-7 (RCB1366) (15) and HEK293T (CRL-11268) cell lines as control cells. All cell lines were cultured in Glutamax $^{\circledR}$-supplemented DMEM containing $10 \%$ fetal bovine serum.

\section{2 | HCV Virus}

We used two recombinant viruses derived from the JFH1 strain and expressing a Renilla luciferase reporter gene, the JFH1-CS-A4-RLuc/TM construct (13) and the JFH1-CS-A4RLuc/QM construct which contain the additional mutation I414T in E2, as compared to the JFH1-CS-A4-RLuc/TM strain.

\subsection{HCVec and HCVpp infection}

HuH-7, SNU-182, SNU-398 and SNU-449 cells were inoculated for 3 hours with JFH1derived constructs expressing the luciferase reporter gene. After inoculation, the medium was changed and cells were cultured for 72 hours. HCV infection was assessed by measuring the luciferase activity with a Berthold CentroXS ${ }^{3}$ LB 960 luminometer. HCV entry in HuH-7, SNU-182, SNU-398, SNU-449 and 293T cells was evaluated using retroviral particles expressing firefly luciferase and pseudotyped with HCV JFH1 E1/E2 glycoproteins (HCVpp) (3). Non-infected cells or cells inoculated with particles lacking envelope protein $(\Delta \mathrm{env})$ were 
100

used as negative controls. Inoculation were performed during 3 hours and luciferase were

101 measured 48 hours later.

102

103

104 negative control.

\section{4 | Quantification of miR-122 expression}

The expression of miR-122-5p (assay ID: 002245) was quantified by RT-qPCR using the TaqMan $^{\circledR}$ Small RNA Assays (Life Technologies), after extraction using the mirVana ${ }^{\mathrm{TM}}$ miRNA Isolation Kit (Life Technologies), according to the manufacturer's instructions. The small nuclear RNA RNU6B (assay ID: 001093) was used as endogenous control. The $\Delta \Delta \mathrm{Ct}$ method was used with $\mathrm{HuH}-7$ cells as calibrator.

2.5 Flow cytomety. The CD81 cell surface expression was assessed by flow cytometry. Cells were trypsinized, centrifuged, resuspended in PBS and stained with isotype control or FITC-coupled anti-CD81 mouse monoclonal antibody (JS-81, BD Pharmingen). Samples were analyzed on a Navios flow cytometer (Beckman Coulter).

2.6 CLDN1, ApoE and miR-122 transductions. Lentiviral pseudoparticles were generated by co-transfection of 293 T cells with plasmids encoding HIV gag-pol, the vesicular stomatitis virus envelope protein G (VSV-G) and the proteins of interest (pWPI-ApoE-BLR, kindly provided by T Pietschmann; pTRIP-Cerulean-CLDN1, kindly provided CM Rice). Cells were transduced by overnight incubation with lentiviral pseudoparticles at $37^{\circ} \mathrm{C}$. Cells were also transduced with miR-122-expressing lentiviral particles (miRIDIAN shMIMIC Lentiviral microRNA, Thermo Scientific) to stably express miR-122 or the non-silencing control lentiviral particles (miRIDIAN shMIMIC Lentiviral microRNA, Thermo Scientific), as 
1252.7 Western blot analysis. Cells were lysed with RIPA buffer. Protein content of cell

126 lysates was determined using the BCA protein assay kit (Pierce). The proteins were 127 precipitated, separated by SDS-PAGE and transferred to Hybond-ECL nitrocellulose

128 membranes (GE Healthcare). Proteins were detected with specific antibodies diluted 1:1,000

129 (polyclonal anti-SR-B1 (Santa Cruz, ref sc-67098), polyclonal anti-Occludin (Invitrogen),

130 polyclonal anti-Claudin 1 (Invitrogen), polyclonal anti-NPC1L1 (Cell Signaling), polyclonal

131 anti-ApoE (AB947 Millipore) and monoclonal anti-actin (C4, Millipore) and secondary

132 antibodies conjugated to peroxidase (Sigma-Aldrich). The immune complexes were

133 visualized by enhanced chemiluminescence detection (ECL; GE Healthcare) as recommended

134 by the manufacturer.

135

2.8 Quantification of HCV core protein and ApoE. Intracellular or secreted HCV core antigen was quantified by a fully automated chemiluminescent microparticle immunoassay (Architect HCVAg; Abbott, Germany), according to the manufacturer's instructions. specific ELISA assay, according to the manufacturer's instructions (Abcam).

2.9 | Statistical analysis. Mann-Whitney test was used to compare the results obtained with

143 the different cell lines to that obtained with HuH-7 cells. Values of $p<0.05$ and $<0.01$ were 144 considered as thresholds for significance.

147 3.1 | SNU-182, SNU-398 and SNU-449 cells are not permissive to HCV entry and replication 

and observed that SNU-182, SNU-398 and SNU-449 cells were not permissive to HCV infection (13). This result indicated that the entry step and/or the replication step could not be achieved in these cells. In order to determine which step was affected, we first assessed HCV entry using $\mathrm{HCVpp}$ and pseudoparticles lacking envelope ( $\triangle$ Envpp) or non-infected cells as negative controls. As shown in Figure 1A, we observed that SNU-182 and SNU-449 cells enable low levels of HCV entry as compared to HuH-7 cells $\left(2.810^{3}, 2.010^{4}\right.$ and $4.210^{5}$ RLU, respectively) while the results obtained with SNU-398 were similar to that of the negative controls $\left(2.210^{2} \mathrm{RLU}\right)$. with a JFH1 replicon expressing a luciferase reporter gene. Similar levels of electroporation

160 were obtained for each cell line, as measured 4 hours after electroporation $(1.5,0.8,0.6$ and $6.910^{5}$ RLU for HuH-7, SNU-182, SNU-398 and SNU-449, respectively (Figure 1B)). In contrast, while HuH-7 cells achieved high levels of HCV replication (2.5 10 $0^{7}$ RLU at $72 \mathrm{~h}$ ) the replication levels reached at $72 \mathrm{~h}$ in SNU-182, SNU-398 and SNU-449 cells were similar to that of the background measured in mock electroporated cells (1.0, 0.9 and $4.210^{3} \mathrm{RLU}$ at $72 \mathrm{~h}$ ). Altogether, these results suggested that both the entry and replication steps of HCV life cycle are blocked in SNU-182, SNU-398 and SNU-449 cells.

3.2 Stable expression of miR-122 partly restored HCV RNA replication in SNU-182,

Deficiency in miR-122 expression has been shown to be responsible for the absence of

171 HCV RNA replication in several cell lines (17-20). For this reason, we assessed the expression of this miRNA by RT-qPCR in SNU-182, SNU-398 and SNU-449 cells. As shown

173 in Figure 2A, we observed that these three cell lines expressed very low level of miR-122 as 
174 compared to $\mathrm{HuH}-7$ cells, at least 1000-fold lower. To investigate whether expression of miR-

175122 could restore HCV RNA replication in these cells, we transduced the miRNA with the

176 help of lentiviruses. Transduction of $293 \mathrm{~T}$ cells were used as positive control. While

177 transduction of a control miRNA (miR-ctrl) had no effect on miR-122 expression, we

178 observed that SNU-182, SNU-398, SNU-449 and 293T cells transduced with miR-122

179 express similar level of miR-122 as $\mathrm{HuH}-7$ cells (Figure 2A). As previously demonstrated, we

180 confirmed that transduction of miR-122 in $293 \mathrm{~T}$ cells permit to reach similar level of HCV

181 RNA replication as in HuH-7 cells (2.2 $10^{7}$ and $4.310^{7}$ RLU at $72 \mathrm{~h}$, respectively; Figure 2B).

182 In contrast, although HCV RNA replication was improved, only low levels were achieved 183 after miR-122 transduction of SNU-182, SNU-398 and SNU-449 cells, $\left(8.510^{5}, 2.410^{5}\right.$ and

$1841.110^{5}$ RLU at $72 \mathrm{~h}$, respectively; Figure 2B). This low level of HCV RNA replication in

185 SNU-182, SNU-398 and SNU-449 transduced cells was confirmed by the detection of 186 intracellular Core protein (Figure 2C). Thus, these results indicate that the expression of miR187122 in SNU-182, SNU-398 and SNU-449 improves the replication of HCV RNA but is not 188 sufficient to achieve high level of replication.

189 Recently, it has also been shown that SEC14L2 enables pan-genotype HCV replication 190 in cell culture (21). We thus investigated whether transduction of SEC14L2 in SNU-182, 191 SNU-398 and SNU-449 cells could improve HCV replication but we did not observe any 192 effect (data not shown). However, this result was predictable since Saeed et al. also 193 mentioned that replication of the JFH-1 strain was not enhanced by SEC14L2. We also 194 wondered whether induction of the innate immune response could be responsible for the low 195 replication level, however we did not detect any induction of Interferon- $\beta$ and Interferon- $\lambda$ 196 mRNA expression after infection (data not shown). This was corroborated by the fact that the 197 use of pyridone-6, an inhibitor of the Janus Kinases involved in the Interferon-mediated 198 signaling, did not increase the replication level (data not shown). 
Finally, since low levels of replication were achieved in SNU-182, SNU-398 and SNU-449 cells transduced with miR-122, we wondered whether they were able to assemble and secrete progeny virions. However, we did not detect any infectious particle production (Figure 2D) and levels of extracellular Core protein remained very low or undetectable (Figure 2C).

\section{3 | CLDN1 transduction improves HCV entry in SNU-182-miR-122 and SNU-398- miR-122 cells}

To understand HCV entry defect in SNU-182, SNU-398 and SNU-449 cells, we evaluated the expression level of several cellular entry factors. Flow cytometry analysis revealed that CD81 is correctly expressed at the surface of each cell line (Figure 3A). In addition, we observed by western blot using specific antibodies that the three cell lines expressed high levels of SR-BI, OCLN and NPC1L1 (Figure 3B). In contrast, CLDN1 was only very slightly detected in SNU-182 cells and undetectable in SNU-398 cells.

Using SNU-182, SNU-398 and SNU-449 cells transduced with miR-122, we investigated whether stable expression of exogenous CLDN1 could improve HCV entry. These cells were transduced with lentiviruses expressing Cerulean-CLDN1 and 293T cells were used as control. The efficiency of the transduction was checked by western blot (Figure 4A) and the effect on HCV entry was evaluated with the help of HCVpp and HCVcc (Figure 4B). These experiments showed that exogenous CLDN1 expression slightly improves HCVpp entry in SNU-182-miR-122 and SNU-398-miR-122 cells as compared to the increase observed in 293T cells (8.3-, 5.7- and 64.2-fold increase respectively, as compared to cells transduced with a control lentivirus). The improvement observed in HCVcc entry was lower, probably because of the low RNA replication level in these cells, as described above. In 
223 HCV entry in SNU-449-miR-122 cells.

\section{6}

addition, our results suggest that Cerulean-CLDN1 transduction did not have any effect on

\section{4 | ApoE transduction improves infectious virus production in SNU-182-miR-122-}

\section{CLDN1 and SNU-398-miR-122-CLDN1 cells}

As shown in Figure 2B and 2D, whereas low levels of HCV RNA replication were achieved in miR-122 transduced SNU-182, SNU-398 and SNU-449 cells, we did not detect any production of progeny virions. Since ApoE is known to be essential for assembly and secretion of new virions, we assessed the expression level of this protein in these cells. Western blot analysis and ELISA assay suggested that ApoE was not expressed in SNU-398miR-122-CLDN1 and SNU-449-miR-122 cells whereas it was marginally expressed and secreted by SNU-182-miR-122-CLDN1 cells. We thus tested whether stable expression of exogenous ApoE could help these cells to produce infectious virions. After transduction, ApoE was readily detectable in cell lysates and cell supernatants of SNU-398-miR-122CLDN1 and SNU-449-miR-122 cells (Figure 5A and 5B). In contrast, while it was readily detected in the cell lysate of SNU-182-miR-122-CLDN1 cells, the concentration in cell supernatant was low, suggesting a potential defect in secretion. Interestingly, we observed that stable expression of ApoE in SNU-182-miR-122-CLDN1 and SNU-398-miR-122-CLDN1 cells gave rise to a production of progeny virions whereas it had no effect on viral production in SNU-449-miR-122 cells (Figure 5C). These results were confirmed by quantification of Core in the cell supernatant (Figure 5D).

\section{4 | DISCUSSION}

In our previous study, we identified three human hepatocellular carcinoma cell lines established from the primary tumors of Korean patients, SNU-182, SNU-398 and SNU-449, 
that are not permissive to HCV (13). For each cell line, we identified the different steps that

249 were compromised and tried to decipher the mechanisms of the impairments (cf summary 250 Table 1).

We first observed that defect in CLDN1 expression was responsible for the inefficiency of the entry step in SNU-182 and SNU-398. We also demonstrated that its ectopic expression render these cells permissive to this step. CLDN1 was identified as an HCV entry factor thanks to a cyclic lentivirus based repackaging screen of a complementary DNA library, derived from the highly HCV-permissive hepatocarcinoma Huh-7.5 cell line, for genes that render the $293 \mathrm{~T}$ cell line permissive to HCVpp (22). For this reason, 293T cells are widely used to perform functional studies on the role of CLDN1 in HCV entry. It has also been shown that CLDN1 expression alone conferred limited permissiveness for HCV infection in these cells and that co-expression of CD81, OCLN and SR-BI enhance HCV entry to a level higher than that in Huh7.5.1 cells (20). However, it has to be kept in mind that many clones of 293T cells exist which may explain some discrepancies between studies.

Other models such as $\mathrm{H} 1 \mathrm{H}$ or HuH6 cells have been used $(23,24)$ and in this study we 263 identified SNU-182 and SNU-398 cells as alternative cell culture models, expressing high 264 levels of CD81, OCLN, SR-BI and NPC1L1, to perform functional studies of the role of CLDN1 in HCV entry.

MiR-122 is an abundant, liver-specific miRNA that is an unusual host factor for HCV. This miRNA binds HCV RNA and slows decay of the viral genome through protection from 2685 5' exonuclease activity of the host mRNA decay machinery (25). Many studies demonstrated 269 that supplementation of several non-permissive cell lines with miR-122 render them 270 permissive for transient replication of HCV RNA (17-20, 26-28). In this study, we observed 271 that miR-122 is expressed at a low level in SNU-182, SNU-398 and SNU-449 cells. We also 272 demonstrated that transduction of this miRNA in these cells improves HCV RNA replication. 
273 However, in contrast to 293T transduced cells, viral replication in SNU-182, SNU-398 and

274 SNU-449 cells remained very low even after transduction and this was not due to induction of 275 innate immune response. It is thus likely that other dependency host factors essential for the 276 replication are lacking. Alternatively, this could be explained by the presence of restriction 277 factors that decrease the efficiency of this step. Our results also suggest that transduction of 278 ApoE permit the production of low level of HCV infectious particles in SNU-182 and SNU279398 cells transduced with miR-122 but not in SNU-449 cells. However, it is difficult to 280 evaluate precisely the efficiency of this step because of the low level of replication. Altogether, our results identified SNU-182, SNU-398 and SNU-449 cells as 282 hepatocarcinoma cell lines that can be used as models for the study of the functional role of 283 CLDN1, miR-122 and ApoE in the entry, replication and assembly step of HCV life cycle. 284 These cell lines could also be used to identify new dependency/restriction host factors essential for the different step of the viral cycle, thanks to screening strategies, as described 286 previously $(21,22,29)$.

Acknowledgements

We thank Catherine Moriset for assistance. We also thank T Pietschmann and CM

292 Rice for providing us with reagents.

\section{Conflicts of interest}

295 The authors declare that there are no conflict of interest. 
Fournier et al. - page 13

298 This study was funded by the "Conseil Regional de Picardie", grant number REG13047

299 "Oncovir".

300

301

302 
1 Murray CL, Rice CM. Turning hepatitis C into a real virus. Annual Review of Microbiology. 2011; 65:307-27.

3072 Lohmann V, Körner F, Koch J-O, Herian U, Theilmann L, Bartenschlager R. 308 Replication of subgenomic hepatitis C virus RNAs in a hepatoma cell line. Science. 1999; 309 285:110-3.

3103 Bartosch B, Dubuisson J, Cosset FL. Infectious hepatitis C pseudo-particles containing 311 functional E1E2 envelope protein complexes. J Exp Med. 2003; 197:633-42.

3124 Hsu M, Zhang J, Flint M, et al. Hepatitis C virus glycoproteins mediate pH-dependent 313 cell entry of pseudotyped retroviral particles. Proc Natl Acad Sci USA. 2003; 100(12):7271-6.

3145 Lindenbach BD, Evans MJ, Syder AJ, et al. Complete replication of hepatitis C virus 315 in cell culture. Science. 2005; 309:623-6.

3166 Wakita T, Pietschmann T, Kato T, et al. Production of infectious hepatitis C virus in 317 tissue culture from a cloned viral genome. Nat Med. 2005; 11(7):791-6.

3187 Douam F, Lavillette D, Cosset FL. The mechanism of HCV entry into host cells. Prog 319 Mol Biol Transl Sci. 2015; 129:63-107.

3208 Jopling CL, Yi M, Lancaster AM, Lemon SM, Sarnow P. Modulation of hepatitis C 321 virus RNA abundance by a liver-specific MicroRNA. Science. 2005; 309(5740):1577-81.

3229 Chang KS, Jiang J, Cai Z, Luo G. Human apolipoprotein E is required for infectivity and production of hepatitis C virus in cell culture. J Virol. 2007; 81(24):13783-93.

10 Jiang J, Luo G. Apolipoprotein E but not B is required for the formation of infectious hepatitis C virus particles. J Virol. 2009; 83(24):12680-91.

11 Benga WJ, Krieger SE, Dimitrova M, et al. Apolipoprotein E interacts with hepatitis C virus nonstructural protein $5 \mathrm{~A}$ and determines assembly of infectious particles. Hepatology. 2010; 51(1):43-53.

12 Cun W, Jiang J, Luo G. The C-terminal alpha-helix domain of apolipoprotein $\mathrm{E}$ is required for interaction with nonstructural protein $5 \mathrm{~A}$ and assembly of hepatitis $\mathrm{C}$ virus. $J$ Virol. 2010; 84(21):11532-41.

13 Helle F, Brochot E, Fournier C, et al. Permissivity of primary human hepatocytes and different hepatoma cell lines to cell culture adapted hepatitis C virus. PLoS ONE. 2013; 8(8):e70809.

14 Park JG, Lee JH, Kang MS, et al. Characterization of cell lines established from human hepatocellular carcinoma. Int J Cancer. 1995; 62(3):276-82.

15 Nakabayashi H, Taketa K, Miyano K, Yamane T, Sato J. Growth of human hepatoma cells lines with differentiated functions in chemically defined medium. Cancer Res. 1982; 42(9):3858-63.

16 Jones CT, Catanese MT, Law LM, et al. Real-time imaging of hepatitis C virus infection using a fluorescent cell-based reporter system. Nat Biotechnol. 2010; 28(2):167-71.

17 Narbus CM, Israelow B, Sourisseau M, et al. HepG2 cells expressing microRNA miR122 support the entire hepatitis C virus life cycle. $J$ Virol. 2011; 85(22):12087-92.

18 Kambara H, Fukuhara T, Shiokawa M, et al. Establishment of a Novel Permissive Cell Line for the Propagation of Hepatitis C Virus by Expression of MicroRNA miR122. J Virol. 2012; 86(3):1382-93.

19 Thibault PA, Huys A, Dhillon P, Wilson JA. MicroRNA-122-dependent and independent replication of Hepatitis C Virus in Hep3B human hepatoma cells. Virology. 2013; 436(1):179-90. 
20 Da Costa D, Turek M, Felmlee DJ, et al. Reconstitution of the entire hepatitis C virus 351 life cycle in nonhepatic cells. $J$ Virol. 2012; 86(21):11919-25.

35221 Saeed M, Andreo U, Chung HY, et al. SEC14L2 enables pan-genotype HCV replication in cell culture. Nature. 2015; 524(7566):471-5.

35422 Evans MJ, von Hahn T, Tscherne DM, et al. Claudin-1 is a hepatitis C virus coreceptor required for a late step in entry. Nature. 2007; 446(7137):801-5.

35623 Cukierman L, Meertens L, Bertaux C, Kajumo F, Dragic T. Residues in a highly conserved claudin-1 motif are required for hepatitis $\mathrm{C}$ virus entry and mediate the formation of cell-cell contacts. $J$ Virol. 2009; 83(11):5477-84.

35924 Haid S, Windisch MP, Bartenschlager R, Pietschmann T. Mouse-specific residues of

360 claudin-1 limit hepatitis $\mathrm{C}$ virus genotype 2 a infection in a human hepatocyte cell line. $J$ Virol. $3612010 ; 84(2): 964-75$.

36225 Sedano CD, Sarnow P. Interaction of host cell microRNAs with the HCV RNA genome during infection of liver cells. Semin Liver Dis. 2015; 35(1):75-80.

36426 Chang J, Guo JT, Jiang D, Guo H, Taylor JM, Block TM. Liver-specific microRNA 365 miR-122 enhances the replication of hepatitis C virus in nonhepatic cells. J Virol. 2008; 366 82(16):8215-23.

36727 Fukuhara T, Kambara H, Shiokawa M, et al. Expression of microRNA miR-122 368 facilitates an efficient replication in nonhepatic cells upon infection with hepatitis $\mathrm{C}$ virus. $J$ 369 Virol. 2012; 86(15):7918-33.

37028 Lin LT, Noyce RS, Pham TN, et al. Replication of subgenomic hepatitis C virus 371 replicons in mouse fibroblasts is facilitated by deletion of interferon regulatory factor 3 and 372 expression of liver-specific microRNA 122. J Virol. 2010; 84(18):9170-80.

37329 Ploss A, Evans MJ, Gaysinskaya VA, et al. Human occludin is a hepatitis C virus entry factor required for infection of mouse cells. Nature. 2009; 457(7231):882-6. 


\section{FIGURE LEGENDS}

383 Figure $1 \mathrm{HCV}$ entry and replication in SNU-182, SNU-398 and SNU-449 cells. A. HuH-7, 384 SNU-182, SNU-398 and SNU-449 cells were inoculated for $4 \mathrm{~h}$ with HCVpp or $\Delta$ envpp to 385 monitor $\mathrm{HCV}$ entry. Luciferase assays were performed $48 \mathrm{~h}$ post-infection. Results are 386 expressed as Relative Light Units (RLU) and are reported as the means \pm S.D. of three 387 independent experiments. B. JFH1 RNA encoding a luciferase reporter gene and deleted for 388 E1E2 region was electroporated in HuH-7, SNU-182, SNU-398 and SNU-449 cells. RNA 389 Replication was assessed by measuring luciferase activity at 4 and $72 \mathrm{~h}$ post-electroporation. 390 Results are expressed as Relative Light Units (RLU) and are reported as the means \pm S.D. of 391 three independent experiments. Differences were considered statistically significant if $p<0.05(*)$ or $p<0.01(* *)$.

Figure 2 Stable expression of miR-122 partly restored HCV RNA replication in SNU-182, SNU-398 and SNU-449 cells. A. The expression of miR-122 was determined by RT-qPCR in HuH-7, SNU-182, SNU-398, SNU-449 and 293T cells, before (NT for non-transduced) or after transduction with lentiviruses expressing a control miRNA (miR-ctrl) or miR-122. The $\Delta \Delta \mathrm{Ct}$ method was used for quantification, with the small nuclear RNA RNU6B as endogenous control and HuH-7 cells as calibrator. All results are representative of 3

400 independent experiments. B. SNU-182, SNU-398, SNU-449 and 293T cells, transduced with 401 miR-ctrl or miR-122, were electroporated with JFH1 RNA encoding a luciferase reporter gene. 402 The replication was assessed by measuring luciferase activities at 4, 24, 48 and $72 \mathrm{~h}$. Results 403 are expressed as RLU and are reported as means \pm S.D. of three independent experiments. C. 404 Core protein concentration was determined in the cell culture supernatants and the cell lysates 405 recovered $72 \mathrm{~h}$ post-electroporation. Results are reported as the means \pm S.D. of three 
406 independent experiments (ND : not determined). D. Infectious particle production was 407 evaluated after inoculation of naive $\mathrm{HuH}-7$ cells with the supernatants recovered from 408 electroporated cells. HuH-7 cells were inoculated during $4 \mathrm{~h}$ and luciferase activities were 409 measured $72 \mathrm{~h}$ post-infection. Results are expressed as RLU and are reported as the means \pm 410 S.D. of three independents experiment. Differences were considered statistically significant if $411 p<0.05(*)$ or $p<0.01(* *)$.

Figure 3 Evaluation of HCV cell entry factor expression in SNU-182, SNU-398 and SNU449 cells. A. Flow cytometry was performed to assess the expression of CD81 in SNU-182, SNU-398, and SNU-449, using FITC-coupled anti-CD81 antibody (grey) or isotype control (white). HuH-7 cells were used as control. B. SR-B1, CLDN1, OCLN and NPC1L1 expression in each cell line was evaluated by western blot. $\beta$-actin served as a loading control. The molecular weight (MW) of each protein is indicated on the left.

Figure 4 Ectopic expression of CLDN1 in SNU-182-miR-122 and SNU-398-miR-122 cells improves HCV entry. A. SNU-182-miR-122, SNU-398-miR-122, SNU-449-miR-122 and $293 \mathrm{~T}$ cells were transduced with lentiviruses expressing Cerulean-CLDN1. Empty lentiviruses were used as negative control. Cerulean-CLDN1 expression was evaluated by 424 western blot. $\beta$-actin served as a loading control. The molecular weight (MW) of each protein 425 is indicated on the left. B. HCV entry in each cell line was evaluated after inoculation with 426 HCVpp or HCVcc for 4 h. 293T cells were used as control. Infection was determined by 427 measuring luciferase activities $72 \mathrm{~h}$ post-infection. Results are expressed as RLU and are 428 reported as the means \pm S.D. of three independent experiments. Differences were considered 429 statistically significant if $p<0.05(*)$ or $p<0.01(* *)$. 
431 Figure 5 Ectopic expression of ApoE enables HCVcc production in the SNU182-miR-122-

432 CLDN-1 and SNU398-miR-122-CLDN-1 cells. A. SNU-182-miR-122-CLDN1, SNU-398433 miR-122-CLDN1 cells and SNU-449-miR-122 cells were transduced with lentiviruses 434 expressing ApoE. HuH-7 and 293T-miR122-CLDN1 cells were used as control. Non435 transduced cells were used as negative control. ApoE expression was evaluated by western 436 blot. $\beta$-actin served as a loading control. The molecular weight (MW) of each protein is 437 indicated on the left. B. The concentration of ApoE secreted in the supernatant of each cell 438 line after $72 \mathrm{~h}$ was evaluated by Elisa assay. Results are reported as the means \pm S.D. of two 439 independent experiments. C. The different cell lines were electroporated with JFH1 RNA 440 encoding a luciferase reporter gene and the supernatants were recovered $72 \mathrm{~h}$ after 441 electroporation. Infectious particle production was evaluated by inoculating naive HuH-7 cells 442 with these supernatants for $4 \mathrm{~h}$ and measuring luciferase activities $72 \mathrm{~h}$ post-infection. Results 443 are expressed as RLU and are reported as the means \pm S.D. of three independent experiments. 444 D. Core protein concentration in the cell culture supernatants and the cell lysates was 445 determined $72 \mathrm{~h}$ post-electroporation. Results are reported as the means \pm S.D. of at least two 446 independent experiments. Differences were considered statistically significant if $p<0.05(*)$ 447 or $p<0.01(* *)$. 
TABLE 1 Features of the cell lines used in this study

\begin{tabular}{|c|c|c|c|c|c|c|c|c|c|c|}
\hline Cell line & Entry & Replication & Assembly & CD81 & SR-BI & CLDN1 & OCLN & NPC1L1 & mir-122 & ApoE \\
\hline $\mathrm{HuH}-7$ & ++ & ++ & ++ & ++ & ++ & ++ & ++ & ++ & ++ & ++ \\
\hline SNU-182 & $+/-$ & - & - & ++ & ++ & $+/-$ & ++ & ++ & - & + \\
\hline SNU-182-miR122 & $+/-$ & + & - & ++ & ++ & $+/-$ & ++ & ++ & ++ & + \\
\hline SNU-182-miR-122-CLDN1 & + & + & - & ++ & ++ & ++ & ++ & ++ & ++ & + \\
\hline SNU-182-miR-122-CLDN1-ApoE & + & + & + & ++ & ++ & ++ & ++ & ++ & ++ & ++ \\
\hline SNU-398 & - & - & - & ++ & ++ & - & ++ & ++ & - & - \\
\hline SNU-398-miR122 & - & + & - & ++ & ++ & - & ++ & ++ & ++ & - \\
\hline SNU-398-miR-122-CLDN1 & + & + & - & ++ & ++ & ++ & ++ & ++ & ++ & - \\
\hline SNU-398-miR-122-CLDN1-ApoE & + & + & + & ++ & ++ & ++ & ++ & ++ & ++ & ++ \\
\hline SNU-449 & + & - & - & ++ & ++ & + & ++ & ++ & - & - \\
\hline SNU-449-miR122 & + & + & - & ++ & ++ & + & ++ & ++ & ++ & - \\
\hline SNU-449-miR-122-CLDN1 & + & + & - & ++ & ++ & ++ & ++ & ++ & ++ & - \\
\hline SNU-449-miR-122-ApoE & + & + & - & ++ & ++ & + & ++ & ++ & ++ & ++ \\
\hline
\end{tabular}


A

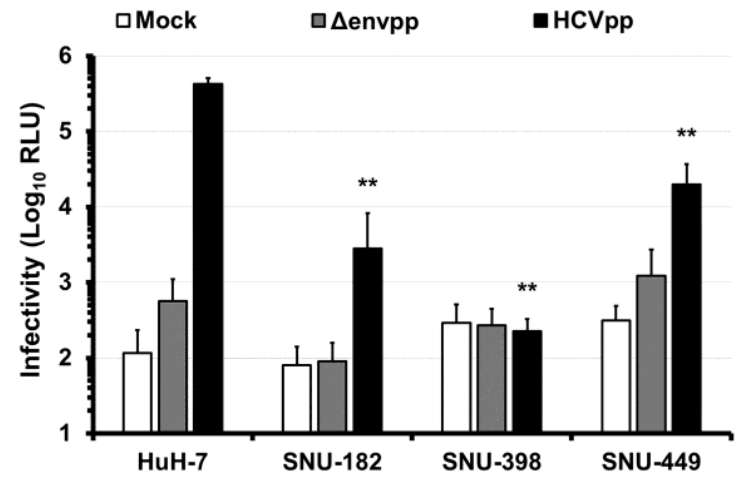

B

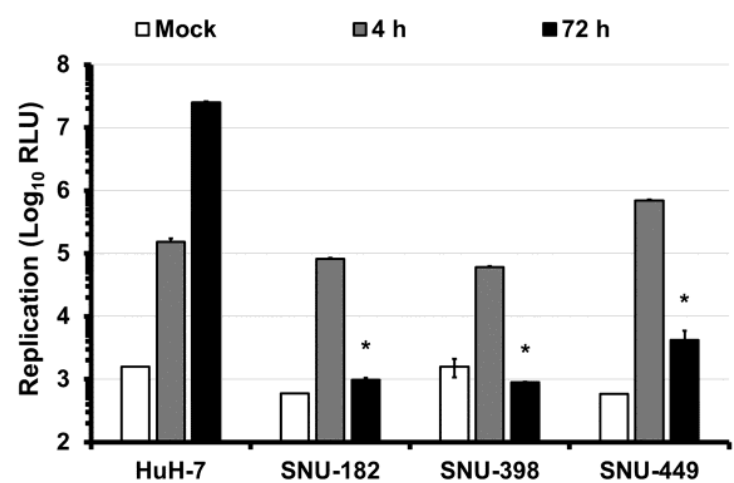

FIG 1 
Fournier et al. - page 21

A

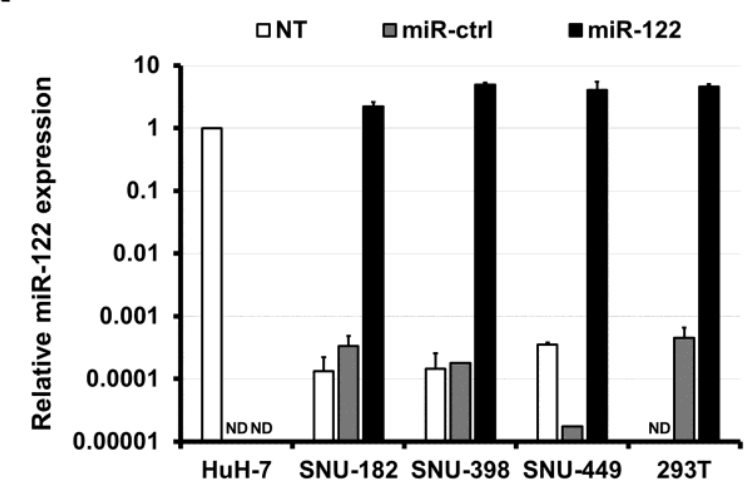

D

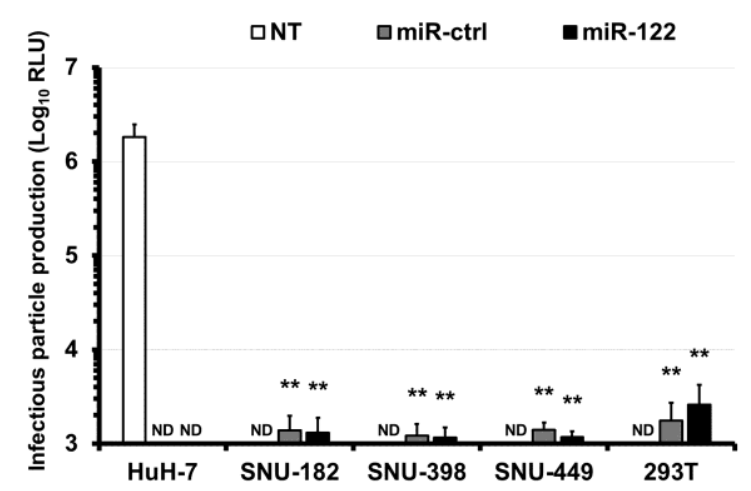

B



C

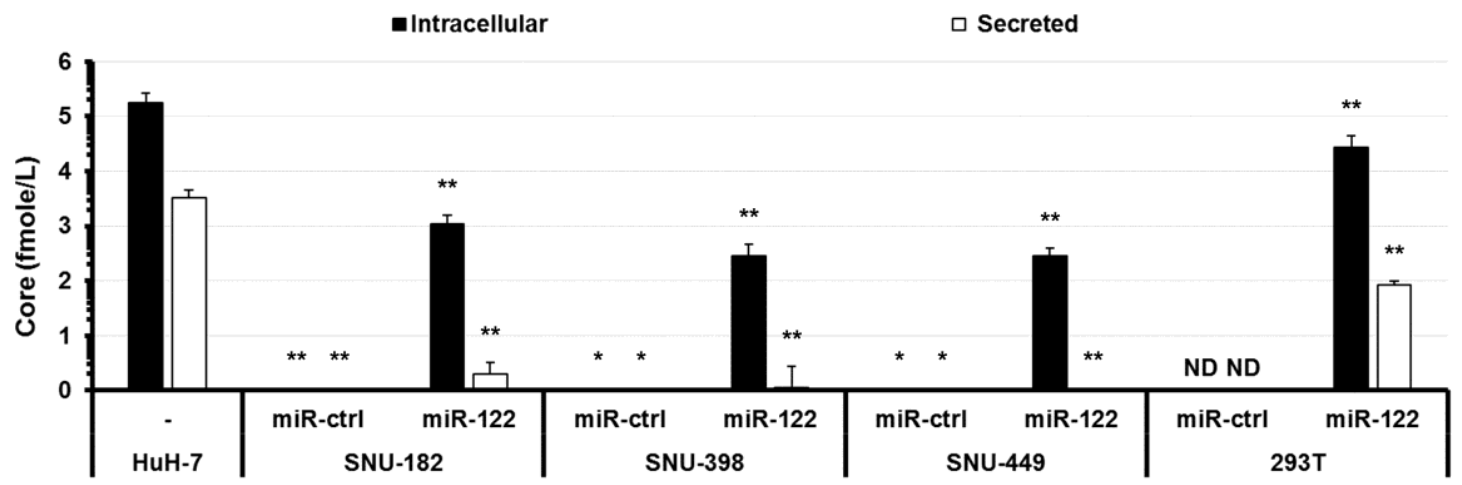

FIG 2 
A

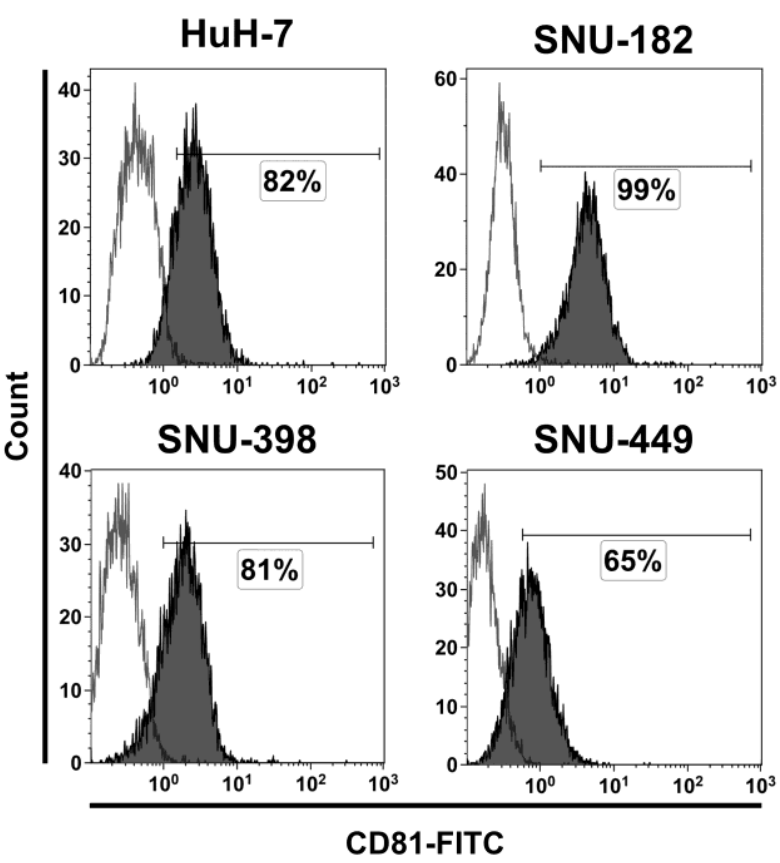

B

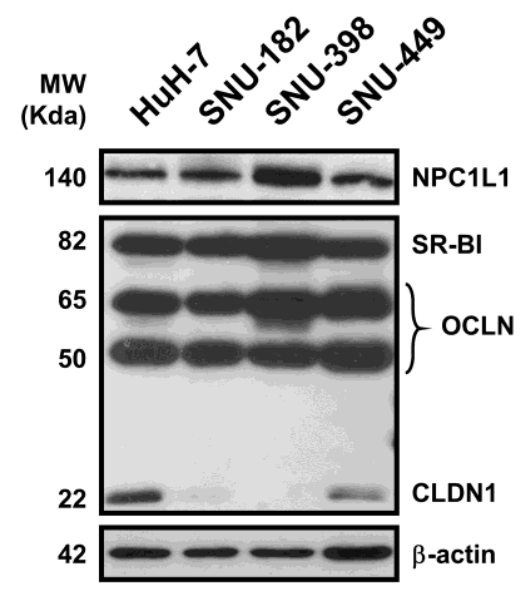

FIG 3 
Fournier et al. - page 23

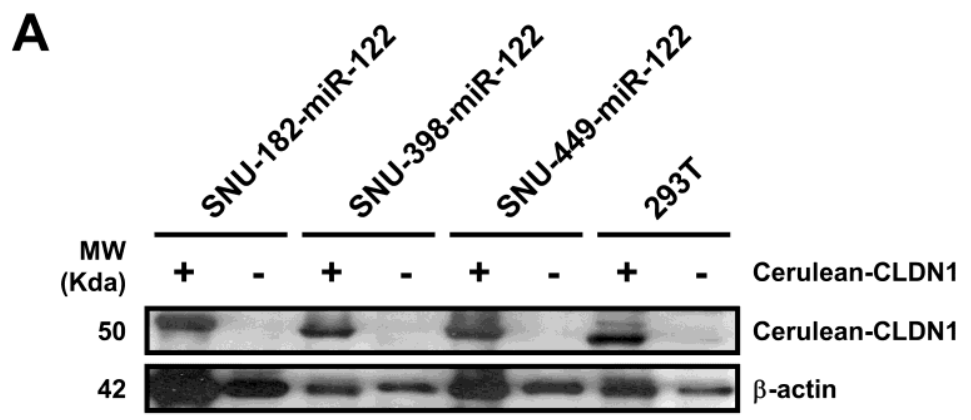

B

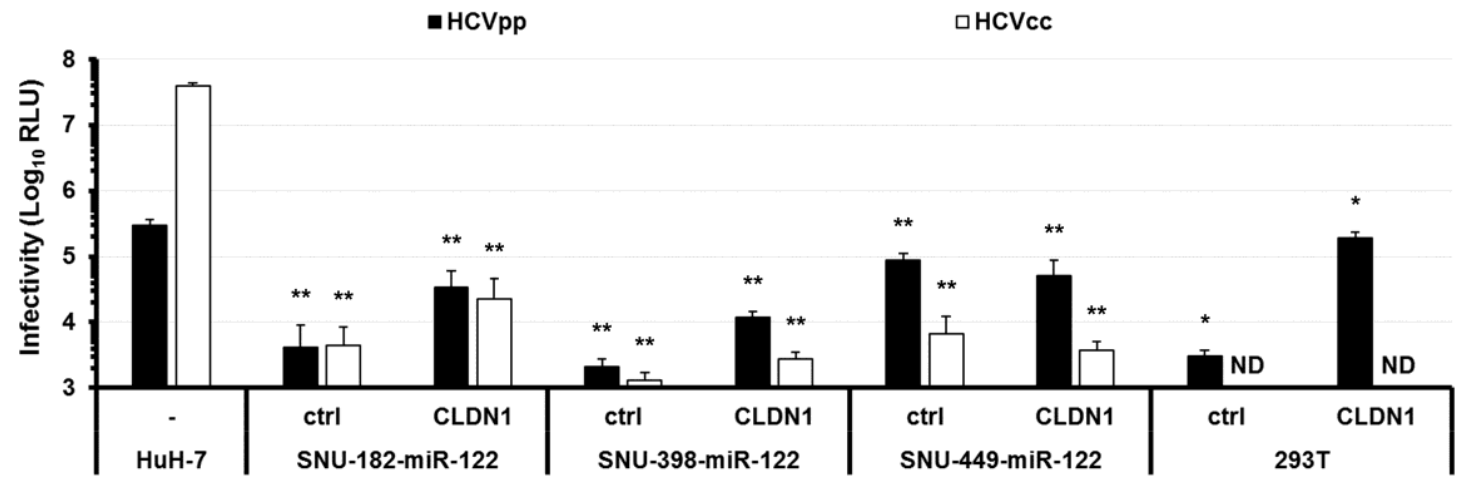

FIG 4 


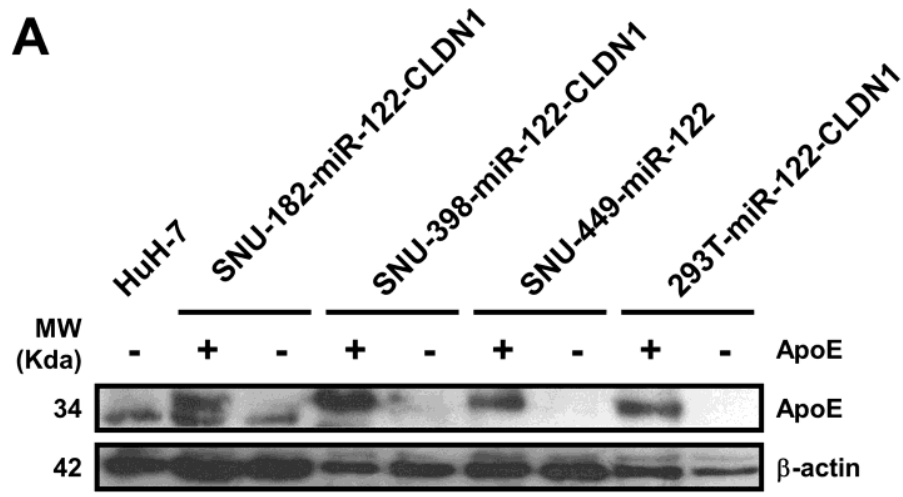

B

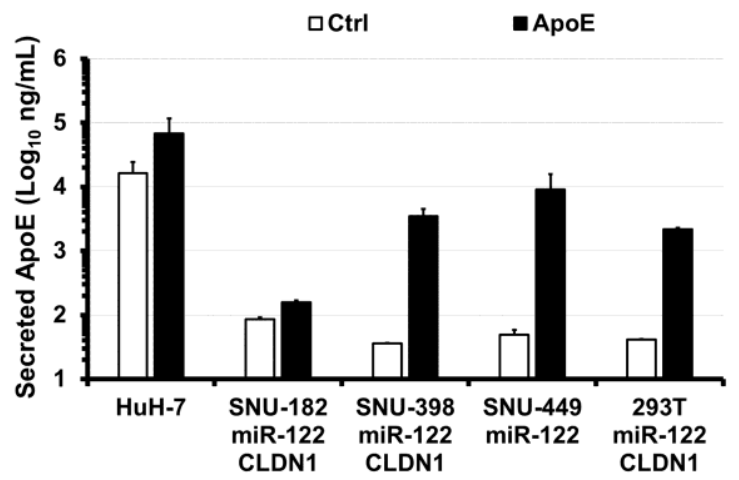

D



FIG 5 